\title{
Asymptotic Error Estimates for Gaussian Quadrature Formulas
}

\author{
By T. H. Charles Chen*
}

\begin{abstract}
This paper gives derivative-free asymptotic error estimates for the Gaussian quadrature formula with the nonnegative weight function $w(x)$ belonging to a certain class. Numerical examples are presented.
\end{abstract}

1. Introduction. Let the nonnegative weight function $w(x)$ be defined on the interval $[-1,1]$ such that the moments $\int_{-1}^{1} w(x) x^{k} d x$ exist for $k=0,1,2, \ldots$ It is well known [3, p. 74] that the following Gaussian quadrature rule has precision $2 n-1$,

$$
\int_{-1}^{1} w(x) f(x) d x=\sum_{k=1}^{n} w_{k} f\left(x_{k}\right)+E_{n}(f)
$$

where $x_{k}, k=1,2, \ldots, n$, are the zeros of the orthonormal polynomial of degree $n$ with respect to the weight function $w(x)$ on the interval $[-1,1]$ and the weights $w_{k}$ are defined by

$$
w_{k}=\int_{-1}^{1} w(x) \frac{P_{n}(x)}{\left(x-x_{k}\right) P_{n}^{\prime}\left(x_{k}\right)} d x .
$$

The following classical representation for the error term $E_{n}(f)$ can be found, e.g., in [3, p. 227],

$$
E_{n}(f)=f^{(2 n)}(\xi)[(2 n) !]^{-1} k_{n}^{-2},
$$

where $-1<\xi<1$ and $k_{n}$ is the leading coefficient of the orthonormal polynomial $P_{n}(x)$ of degree $n$ corresponding to the weight function $w(x)$ on $[-1,1]$. The above representation for $E_{n}(f)$ is of little practical value, since the derivatives $f^{(2 n)}$ are usually too difficult to obtain. In [2], Chawla and Jain have shown derivative-free asymptotic error estimates for the Gauss-Legendre rule, i.e., for the special case $w(x)=1$. The Gauss-Legendre rule can be adversely affected by the nearby singularities of the integrand. In this case we may speed up the convergence by absorbing the critical factors into the weight function $w(x)$; for example, consider $w(x)=\left(x^{2}+a^{2}\right)^{-1}$ in (1) for small $a>0$. Kumar [8], [9] has derived Gaussian quadratures for certain nonclassical weight functions and also given error estimates. Recently several nonclassical $x_{k}$ and $w_{k}$ have been tabulated, e.g., Stroud

Received October 6, 1980; revised February 17, 1981.

1980 Mathematics Subject Classification. Primary 65D30, 65D32; Secondary 41A60.

* Current address: Mathematics Department, The University of Alabama in Huntsville, Huntsville, Alabama 35899. 
and Secrest [11], Piessens and Branders [10]. The main purpose of this paper is to extend Chawla and Jain's results [2] to some nonclassical weight functions $w(x)$ belonging to a certain class.

2. The Function $D\left(\xi^{-1} ; w\right)$. Define

$$
Q_{n}(z)=\int_{-1}^{1} w(x) \frac{P_{n}(x)}{z-x} d x,
$$

for $z \notin[-1,1]$. The error term $E_{n}(f)$ in (1) can be written as

$$
E_{n}(f)=\frac{1}{2 \pi i} \int_{C} \frac{Q_{n}(z)}{P_{n}(z)} f(z) d z
$$

see, e.g., Davis and Rabinowitz [3]. Here $C$ is a simple closed contour in the complex plane and contains the interval $[-1,1]$ in its interior. The function $f(z)$ is assumed to be analytic inside $C$. Following Szegö [12], the functions $w(x)$ and $|\log w(x)|$ are supposed Riemann integrable on $[-1,1]$. In the following theorem, we employ the mapping $z=\frac{1}{2}\left(\xi+\xi^{-1}\right)$ which maps the circle $|\xi|=\rho>1$ in the $\xi$-plane onto the ellipse $E_{\rho}$ in the $z$-plane with foci at $z= \pm 1$ and semiaxes $\frac{1}{2}\left(\rho \pm \rho^{-1}\right)$. For the inverse mapping defined by $\xi=z+\left(z^{2}-1\right)^{1 / 2}$, we choose the sign of square root that makes $|\xi|>1$ for $z \notin[-1,1]$. The following theorem can be found in Barrett [1]:

THEOREM 1. Uniformly on any compact subset of $C /[-1,1]$,

$$
\frac{Q_{n}(z)}{P_{n}(z)}=2 \pi \xi^{-2 n-1} D\left(\xi^{-1} ; w\right)(1+o(1)), \text { as } n \rightarrow \infty,
$$

where $|\xi|>1$ and $z=\left(\xi+\xi^{-1}\right) / 2$. The function $D\left(\xi^{-1} ; w\right)$ is defined by

$$
D\left(\xi^{-1} ; w\right)=\exp \left\{\frac{1}{2 \pi} \int_{-\pi}^{\pi} \log [w(\cos \theta)] \frac{1+\xi^{-1} e^{-i \theta}}{1-\xi^{-1} e^{-i \theta}} d \theta\right\}
$$

for $|\xi|>1$.

Freud [5] and von Sydow [13] have employed the function $D\left(\xi^{-1} ; w\right)$ to find upper bounds for Gaussian quadrature errors. The function $D\left(\xi^{-1} ; w\right)$ has the following algebraic properties,

(i) $D\left(\xi^{-1} ; w_{1} w_{2}\right)=D\left(\xi^{-1} ; w_{1}\right) \cdot D\left(\xi^{-1} ; w_{2}\right)$,

(ii) $D\left(\xi^{-1} ; w_{1} / w_{2}\right)=D\left(\xi^{-1} ; w_{1}\right) / D\left(\xi^{-1} ; w_{2}\right)$,

(iii) $D\left(\xi^{-1} ; w^{p}\right)=D\left(\xi^{-1} ; w\right)^{p}$ for constant $p$,

(iv) $D\left(\xi^{-1} ; k\right)=k$ for constant $k$, and

(v) $D\left(\xi^{-1} ; k w_{1}\right)=k D\left(\xi^{-1} ; w_{1}\right)$ for constant $k$.

The evaluation or estimation of the kernel function $D\left(\xi^{-1} ; w\right)$ depends on the nature of the weight function. Since

$$
\frac{1+\xi^{-1} e^{-i \theta}}{1-\xi^{-1} e^{-i \theta}}=1+2 \sum_{k=1}^{\infty} \xi^{-k} e^{-i k \theta}
$$

we may write (4) in the form

$$
\begin{aligned}
D\left(\xi^{-1} ; w\right)= & \exp \left\{\pi^{-1} \int_{0}^{\pi} \log [w(\cos \theta)] d \theta\right\} \\
& \cdot \exp \left\{2 \pi^{-1} \sum_{k=1}^{\infty} \int_{0}^{\pi} \log [w(\cos \theta)] \cos k \theta d \theta \cdot \xi^{-k}\right\} .
\end{aligned}
$$


If the closed forms for $\int_{0}^{\pi} \log [w(\cos \theta)] \cos k \theta d \theta, k=0,1,2, \ldots$, are known, then we can evaluate $D\left(\xi^{-1} ; w\right)$ exactly. For example, in the Gauss-Jacobi quadrature rule which has the weight function $w(x)=(1+x)^{\alpha}(1-x)^{\beta}, \alpha>-1, \beta>-1$, the function $D\left(\xi^{-1} ; w\right)$ can be written as

$$
D\left(\xi^{-1} ; w\right)=2^{-\alpha-\beta}\left(1+\xi^{-1}\right)^{2 \alpha}\left(1-\xi^{-1}\right)^{2 \beta},
$$

or $D\left(\xi^{-1} ; w\right)=1$, if $\alpha=\beta=0$. For the latter case, we can estimate $E_{n}(f)$ by

$$
E_{n}(f) \simeq-i \int_{C} f(z)\left(z+\left(z^{2}-1\right)^{1 / 2}\right)^{-2 n-1} d z
$$

where the branch of $\left(z^{2}-1\right)^{1 / 2}$ is chosen as before such that $\left|z+\left(z^{2}-1\right)^{1 / 2}\right|>1$ for $z \notin[-1,1]$. This is the representation for the Gauss-Legendre quadrature error previously considered by Chawla and Jain [2]. Closed forms for $\int_{0}^{\pi} \log [w(\cos \theta)] \cos k \theta d \theta, k=1,2, \ldots$, can be obtained in the following case:

LEMMA 1. Assume the following conditions are satisfied:

(i) $w(x) \neq 0$ at $x=1$ and -1 ,

(ii) $w^{\prime} / w$ is a meromorphic function having only (finitely or infinitely many) simple poles at $\alpha_{j} \notin[-1,1]$,

(iii) $\left|w^{\prime}(z) / w(z)\right| \leqslant O\left(\rho^{-1}\right)$ for $z \in E_{\rho}$.

Then

$$
\int_{0}^{\pi} \log [w(\cos \theta)] \cos k \theta d \theta=-\pi k^{-1} \sum_{j} \xi_{\alpha_{j}}^{-k} \underset{z=\alpha_{j}}{\operatorname{Res}_{j}}\left[\frac{w^{\prime}(z)}{w(z)}\right],
$$

for $k=1,2, \ldots$, where $\xi_{\alpha_{j}}=\alpha_{j}+\left(\alpha_{j}^{2}-1\right)^{1 / 2}$ and $\left|\xi_{\alpha_{j}}\right|>1$.

Proof. Choose an ellipse so that $E_{\rho}$ contains $[-1,1]$ in its interior and is contained in a simply connected region in which $w^{\prime} / w$ is analytic. By the Cauchy integral formula we have

$$
\frac{w^{\prime}(x)}{w(x)}=(2 \pi i)^{-1} \int_{E_{\rho}} \frac{w^{\prime}(z)}{w(z)(z-x)} d z
$$

for $x \in[-1,1]$. Employing the technique of integration by parts, we may write

$$
\begin{array}{r}
\int_{0}^{\pi} \log [w(\cos \theta)] \cos k \theta d \theta=k^{-1} \int_{-1}^{1} \frac{w^{\prime}(x)}{w(x)} U_{k-1}(x)\left(1-x^{2}\right)^{1 / 2} d x \\
=(2 \pi k i)^{-1} \int_{E_{\rho}} \frac{w^{\prime}(z)}{w(z)}\left[\int_{-1}^{1}\left(1-x^{2}\right)^{1 / 2} U_{k-1}(x)(z-x)^{-1} d x\right] d z .
\end{array}
$$

We denote by $T_{k}(x), U_{k}(x)$ the classical Chebyshev polynomials of the first and second kind, respectively. Since

$$
U_{n-1}(x)=\left(1-x^{2}\right)^{-1}\left[x T_{n}(x)-T_{n+1}(x)\right]
$$

and

$$
T_{n+1}(x)=2 x T_{n}(x)-T_{n-1}(x)
$$

we may write

$$
\begin{aligned}
\int_{-1}^{1}\left(1-x^{2}\right)^{1 / 2} & U_{k-1}(x)(z-x)^{-1} d x \\
= & \frac{1}{2} \int_{-1}^{1}\left[T_{k-1}(x)-T_{k+1}(x)\right]\left(1-x^{2}\right)^{-1 / 2}(z-x)^{-1} d x .
\end{aligned}
$$


In $[4$, p. 275], Elliott has shown that

$$
\int_{-1}^{1} T_{n}(x)\left(1-x^{2}\right)^{-1 / 2}(z-x)^{-1} d x=\pi\left(z^{2}-1\right)^{-1 / 2}\left(z+\left(z^{2}-1\right)^{1 / 2}\right)^{-n},
$$

where $z \notin[-1,1]$ and $\left|z+\left(z^{2}-1\right)^{1 / 2}\right|>1$. Therefore

$$
\int_{0}^{\pi} \log [w(\cos \theta)] \cos k \theta d \theta=(2 k i)^{-1} \int_{E_{\rho}}\left[w^{\prime}(z) / w(z)\right] \cdot\left(z+\left(z^{2}-1\right)^{1 / 2}\right)^{-k} d z
$$

Without loss of generality, we may arrange the simple poles of $w^{\prime} / w$ in an increasing order in magnitude, i.e., $\left|\alpha_{1}\right| \leqslant\left|\alpha_{2}\right| \leqslant \ldots$. For each simple pole of $w^{\prime} / w, \alpha_{j}$, we increase the value of $\rho$ through a discrete set, $\rho_{1} \leqslant \rho_{2}<\cdots<\rho_{N}$ $<\cdots$, so that each $E_{\rho_{N}}$ contains $\alpha_{j}, j=1,2, \ldots, a(N)$, in its interior and no pole of $w^{\prime} / w$ is on any of the ellipses $\left\{E_{\rho_{j}}\right\}_{j=1}^{\infty}$. For each $j$, let the ellipse $E_{\beta_{j}}$ be described in the positive sense and joined by a cross-cut (not intersecting the interval $[-1,1])$ to a small circle, $\Gamma_{j}$, with center at $\alpha_{j}$ and described in the negative sense. Under condition (iii) we have

$$
\int_{0}^{\pi} \log [w(\cos \theta)] \cos k \theta d \theta=-\pi k^{-1} \sum_{j} \xi_{\alpha_{j}}^{-k} \underset{z=\alpha_{j}}{\operatorname{Res}}\left[\frac{w^{\prime}(z)}{w(z)}\right] .
$$

Applying the above lemma to (5), we obtain

THEOREM 2. Under the assumptions in the above lemma, we have

$$
D\left(\xi^{-1} ; w\right)=\exp \left(A_{0}\right) \cdot \prod_{j}\left(1-\xi_{\alpha_{j}}^{-1} \xi^{-1}\right)^{\gamma_{j}} \text { for }|\xi|>1,
$$

where

$$
A_{0}=\pi^{-1} \int_{-1}^{1} \log [w(x)] /\left(1-x^{2}\right)^{1 / 2} d x
$$

and

$$
\gamma_{j}=2 \underset{z=\alpha_{j}}{\operatorname{Res}}\left[\frac{w^{\prime}(z)}{w(z)}\right]
$$

The importance of the above theorem is that condition (ii) of Lemma 1 can be satisfied by various classes of weight functions $w(x)$ and then $\gamma_{j}$ can be easily computed.

In the above theorem, if $w^{\prime} / w$ has infinitely many simple poles, say $\left|\alpha_{1}\right| \leqslant\left|\alpha_{2}\right|$ $\leqslant \ldots \leqslant\left|\alpha_{N}\right| \leqslant \ldots$, we may use condition (iii) of Lemma 1 to estimate $D\left(\xi^{-1} ; w\right)$ as follows

$$
D\left(\xi^{-1} ; w\right)=\exp \left(A_{0}\right) \prod_{j=1}^{N}\left(1-\xi_{\alpha_{j}}^{-1} \xi^{-1}\right)^{\gamma_{j}} \cdot \exp \left(R_{N}\right) \quad \text { for }|\xi|>1,
$$

where

$$
\left|R_{N}\right| \leqslant 4\left(\rho_{0}^{2}-1\right)^{-1}(N+1)^{-1} \rho_{0}^{-2 N-1}, \quad \xi_{\alpha_{j}}=\alpha_{j}+\left(\alpha_{j}^{2}-1\right)^{1 / 2}, \quad\left|\xi_{\alpha j}\right|>1,
$$

and the ellipse $E_{\rho_{0}}$ contains $\alpha_{1}, \alpha_{2}, \ldots, \alpha_{N}$, and no other $\alpha_{j}$, in its interior. 
If the weight function can be written as $w(x)=(1+x)^{m}(1-x)^{n} w_{1}(x)$ for some constants $m>0, n>0$, and $w_{1}( \pm 1) \neq 0$, then, appealing to the algebraic properties of the function $D\left(\xi^{-1} ; w\right)$ and $(6)$, we have

$$
D\left(\xi^{-1} ; w\right)=2^{-m-n}\left(1+\xi^{-1}\right)^{2 m}\left(1-\xi^{-1}\right)^{2 n} D\left(\xi^{-1} ; w_{1}\right) .
$$

Then we can apply Theorem 2 to the weight function $w_{1}(x)$, in place of $w(x)$.

The definite integral in (7) does not always have a closed form. However, it can be estimated by the Gauss-Chebyshev quadrature rule, i.e., by absorbing the factor $\left(1-x^{2}\right)^{-1 / 2}$ into the weight function; see, for example, [7, p. 115].

In the following special cases, closed forms for the integral in (7) can be found in [6].

COROLlARY 1. If $w(x)=\left(x^{2}+a^{2}\right)^{-1}$ for $a>0$, then

$$
D\left(\xi^{-1} ; w\right)=4\left[a+\left(a^{2}+1\right)^{1 / 2}\right]^{-2}\left\{1+\left[\left(a^{2}+1\right)^{1 / 2}-a\right]^{2} / \xi^{2}\right\}^{-2} .
$$

COROLlaRY 2. If $w(x)=(x+a)^{-1}$ for real $|a|>1$, then

$$
D\left(\xi^{-1} ; w\right)=2\left(a+\left(a^{2}-1\right)^{1 / 2}\right)^{-1}\left(1+\left(a-\left(a^{2}-1\right)^{1 / 2}\right) / \xi\right)^{-2} .
$$

Applying the algebraic properties of the function $D\left(\xi^{-1} ; w\right)$, we can use the above evaluation for the function $D\left(\xi^{-1} ; w\right)$ to obtain error estimates for the nonclassical Gaussian quadrature formulas tabulated by Piessens and Branders in [10, Tables vi and vii] for the weight functions $w(x)=\left(x^{2}+a^{2}\right)^{\alpha}$ and $w(x)=$ $(x+b)^{\alpha}$ for several different values of $a, b$, and $\alpha$.

3. Asymptotic Estimates for $E_{n}(f)$. Substituting (3) into (2), we obtain

$$
E_{n}(f) \simeq-i \int_{C} f(z) \xi^{-2 n-1} D\left(\xi^{-1} ; w\right) d z
$$

The estimation of the above contour integral depends on the nature of the function $f(z)$. We will mimic the techniques previously employed by Elliott [4] and Chawla and Jain [2] by considering the following four cases.

Case 1. $f$ is an entire function. For an entire function $f$, the contour $C$ in (10) can be displaced freely in the complex plane, provided it never crosses the interval $[-1,1]$. We can therefore use the method of steepest descents to estimate $E_{n}(f)$ when the contour is deformed to pass through the saddle points of the integrand. We write (10) as

$$
E_{n}(f) \simeq-i \int_{C} \exp (h(z)) D\left(\xi^{-1} ; w\right) d z
$$

where $h(z)=\log (f(z))-(2 n+1) \log \left(z+\left(z^{2}-1\right)^{1 / 2}\right)$. If $h^{\prime}(z)$ has a simple zero at $z_{0} \notin[-1,1]$ and letting $\xi_{0}=z_{0}+\left(z_{0}^{2}-1\right)$, then we may use the saddle-point method to estimate the above integral. As before, we choose the branch of $\left(z_{0}^{2}-1\right)^{1 / 2}$ such that $\left|\xi_{0}\right|>1$. For large $n$, assuming that the main contribution to the contour integral comes from the portion of the integral passing through the saddle point $z_{0}$ (where $h^{\prime}\left(z_{0}\right)=0$ ), we obtain the following estimate for $E_{n}(f)$ :

$$
E_{n}(f) \approx-i(2 \pi)^{1 / 2} \alpha\left|h^{\prime \prime}\left(z_{0}\right)\right|^{-1 / 2} \exp \left(h\left(z_{0}\right)\right) D\left(\xi_{0}^{-1} ; w\right),
$$


where $|\alpha|=1$ and $\arg (\alpha)=\pi / 2-\frac{1}{2} \arg \left(h^{\prime \prime}\left(z_{0}\right)\right)$. If the weight function $w(x)$ satisfies the conditions in Lemma 1, then using Theorem 2 we can estimate $E_{n}(f)$ as follows:

$$
\begin{aligned}
E_{n}(f) \approx & -i(2 \pi)^{1 / 2} \alpha\left|h^{\prime \prime}\left(z_{0}\right)\right|^{-1 / 2} \exp \left(h\left(z_{0}\right)\right) \exp \left(A_{0}\right) \\
& \cdot \prod_{j}\left(1-\xi_{\alpha_{j}}^{-1} \xi_{0}^{-1}\right)^{\gamma_{j}}
\end{aligned}
$$

where $A_{0}$ is defined in (7), $\alpha_{j}$ are simple poles of $w^{\prime} / w, \gamma_{j}=2 \operatorname{Res}_{z=\alpha_{j}}\left(w^{\prime} / w\right)$, $\xi_{\alpha_{j}}=\alpha_{j}+\left(\alpha_{j}^{2}-1\right)^{1 / 2}$ and $\left|\xi_{\alpha_{j}}\right|>1$.

Example 1. Let $\alpha=.01$ and consider the following Gaussian quadrature rule:

$$
\int_{-1}^{1} \exp (x) /\left(x^{2}+a^{2}\right) d x=\sum_{k=1}^{n} w_{k} \exp \left(x_{k}\right)+E_{n}(f) .
$$

The weights $w_{k}$ and abscissas $x_{k}$ corresponding to the weight function $w(x)=$ $\left(x^{2}+.0001\right)^{-1}$ are available in [10]. Combining (8) and (11), we obtain

$$
\begin{aligned}
E_{n}(f) \approx & 4\left(a+\left(a^{2}+1\right)^{1 / 2}\right)^{-2}(2 \pi)^{1 / 2} \exp \left(z_{0}\right) \xi_{0}^{-2 n-1}\left(z_{0}\right)^{-1 / 2} \\
& \cdot\left\{1+\left[\left(1+a^{2}\right)^{1 / 2}-a\right]^{2} \xi_{0}^{-2}\right\}^{-2}
\end{aligned}
$$

where $z_{0}=\left((2 n+1)^{2}+1\right)^{1 / 2}, \xi_{0}=z_{0}+\left(z_{0}^{2}-1\right)^{1 / 2}$, and $h^{\prime \prime}\left(z_{0}\right)=z_{0}(2 n+1)^{-2}$. In this example, there are two saddle points $\pm\left((2 n+1)^{2}+1\right)^{1 / 2}$. However, the contribution from the negative saddle point is relatively small and can be neglected for our purposes.

TABLE 1

Values for $\left|E_{n}(f)\right|$ in Example 1

\begin{tabular}{lll}
\hline$n$ & actual & estimated \\
\hline 3 & $.2474(-3)$ & $.2762(-3)$ \\
4 & $.1132(-5)$ & $.1227(-5)$ \\
5 & $.3186(-8)$ & $.3396(-8)$ \\
6 & $.6091(-11)$ & $.6413(-11)$ \\
\hline
\end{tabular}

In Table 1, values in parentheses indicate the power of 10 by which the tabulated values are to be multiplied.

In order to weaken the effect of nearby singularities, Kumar [8], [9] has investigated the Gaussian rule (1) with the weight function $w(x)$ replaced by

$$
w(x) /\left[(2 a+1) x^{2}+a^{2}\right]
$$

for $a>0$; where $w(x)=\left(1-x^{2}\right)^{-1 / 2},\left(1-x^{2}\right)^{1 / 2}$, and $[(1-x) /(1+x)]^{1 / 2}$.

Case 2. $f(z)$ has simple poles. Suppose that $f(z)$ has simple poles at $\alpha_{j}, j=$ $1,2, \ldots$ By deforming the closed contour $E_{\rho}$ as in Lemma 1, we have

$$
E_{n}(f) \approx-2 \pi \sum_{j} D\left(\xi_{j}^{-1} ; w\right) \xi_{j}^{-2 n-1} \underset{z=\alpha_{j}}{\operatorname{Res}}[f(z)]
$$

where $\xi_{j}=\alpha_{j}+\left(\alpha_{j}^{2}-1\right)^{1 / 2}$ and $\left|\xi_{j}\right|>1$. For the evaluation or estimation of the function $D\left(\xi^{-1} ; w\right)$, we refer to Theorem 2 . 
Example 2. Consider the Gaussian quadrature error

$$
E_{n}(f)=\int_{-1}^{1} f(x) /\left(x^{2}+a^{2}\right) d x-\sum_{k=1}^{n} w_{k} f\left(x_{k}\right)
$$

where $a=.01$ and $f(x)=\left(x^{2}+1\right)^{-1}$. The integrand $f(x)$ has two simple poles at $\pm i$. Combining (8) and (12), we obtain

$$
\begin{aligned}
E_{n}(f) \approx & (-1)^{n+1} 8 \pi\left(a+\left(a^{2}+1\right)^{1 / 2}\right)^{-2}\left\{1-\left[\left(1+a^{2}\right)^{1 / 2}-a\right]^{2} /(3+2 \sqrt{2})\right\}^{-2} \\
& \cdot(1+\sqrt{2})^{-2 n-1}
\end{aligned}
$$

TABLE 2

Values for $\left|E_{n}(f)\right|$ in Example 2

\begin{tabular}{lll}
\hline$n$ & actual & estimated \\
\hline 3 & $.6887(-1)$ & $.7448(-1)$ \\
4 & $.1219(-1)$ & $.1278(-1)$ \\
5 & $.2109(-2)$ & $.2193(-2)$ \\
6 & $.3644(-3)$ & $.3762(-3)$ \\
\hline
\end{tabular}

Case 3. $f(z)$ has an algebraic singularity on the real axis. Say $f(z)=(c-z)^{\phi} g(z)$, where $c>1, g(z)$ is entire and $\phi$ is rational and nonintegral. To estimate the contour integral (10), we choose the contour as in the proof of Lemma 1, except that the cross-cut now encloses the part of the real axis between the small circle centered at $z=c$, and the ellipse $E_{\rho}$. Again we assume that the integral (10) along $E_{\rho}$ tends to zero as $\rho \rightarrow \infty$. We also assume that $\phi>-1$, so that the integral around the small circle tends to zero. In the limit, therefore, the only contributions to the contour integral (10) come from the line segment of the cross-cut, which combine to give

$$
E_{n}(f) \approx-2 \sin (\pi \phi) \lim _{a \rightarrow \infty} \int_{c}^{a}|c-x|^{\phi} g(x) D\left(\xi^{-1} ; w\right) /\left(x+\left(x^{2}-1\right)^{1 / 2}\right)^{2 n+1} d x
$$

The above integral can be estimated as in [2] and [4]. Assuming that the major contribution to the integral comes from values close to $z=c$, we obtain the estimate

$$
\begin{aligned}
& E_{n}(f) \approx-2 \sin (\pi \phi)\left(c^{2}-1\right)^{(\phi+1) / 2} g(c) D\left(\xi_{c}^{-1} ; w\right) \\
& \cdot(2 n+1)^{-\phi-1} \Gamma(\phi+1) \xi_{c}^{-2 n-1},
\end{aligned}
$$

where $\xi_{c}=c+\left(c^{2}-1\right)^{1 / 2}$ and $\left|\xi_{c}\right|>1$.

Example 3. Consider the Gaussian quadrature error

$$
\int_{-1}^{1} w(x)(2-x)^{1 / 2} d x=\sum_{k=1}^{n} w_{k}\left(2-x_{k}\right)^{1 / 2}+E_{n}(f)
$$


where $w(x)=(x+a)^{-1}, a=1.01$, and $f(x)=(2-x)^{1 / 2}$. The abscissas $x_{k}$ and weights $w_{k}$ are available in [10]. The following explicit form for this integral can be obtained by the substitution $2-x=y^{2}$ :

$$
\begin{aligned}
& \int_{-1}^{1}(2-x)^{1 / 2} /(x+a) d x \\
& \quad=2\left(1-3^{1 / 2}\right)-b \log \left[\left(3^{1 / 2}-b\right)\left(3^{1 / 2}+b\right)^{-1}(1+b)(1-b)^{-1}\right],
\end{aligned}
$$

where $b=(2+a)^{1 / 2}$. Equations (9) and (13) give

$$
\begin{aligned}
E_{n}(f) \approx & -2(27)^{1 / 4} \pi^{1 / 2}\left(a+\left(a^{2}-1\right)^{1 / 2}\right)^{-1}\left\{1+\left[a-\left(a^{2}-1\right)^{1 / 2}\right] / \xi_{c}\right\}^{-2} \\
\cdot & (2 n+1)^{-3 / 2} \xi_{c}^{-2 n-1},
\end{aligned}
$$

where $\xi_{c}=2+3^{1 / 2}$.

TABLE 3

Values for $\left|E_{n}(f)\right|$ in Example 3

\begin{tabular}{lll}
\hline$n$ & actual & estimated \\
\hline 3 & $.3517(-4)$ & $.2472(-4)$ \\
4 & $.1590(-5)$ & $.1218(-5)$ \\
5 & $.8020(-7)$ & $.6470(-7)$ \\
6 & $.4327(-8)$ & $.3616(-8)$ \\
\hline
\end{tabular}

Case 4. $f(z)$ has a logarithmic singularity on the real axis. Consider $f(z)=$ $g(z) \log (c-z)$, where $c>1$, and assume $g(z)$ entire. Since the integral (10) around the small circle centered at $c$ tends to zero as the radius of the circle tends to zero, the contributions from the cross-cut in this case combine to give

$$
E_{n}(f) \approx-2 \pi \lim _{a \rightarrow \infty} \int_{c}^{a} D\left(\xi^{-1} ; w\right)\left(x+\left(x^{2}-1\right)^{1 / 2}\right)^{-2 n-1} g(x) d x .
$$

Applying the same techniques as in Case 3, we obtain the following estimate

$$
E_{n}(f) \approx-2 \pi g(c)\left(c^{2}-1\right)^{1 / 2} D\left(\xi_{c}^{-1} ; w\right)(2 n+1)^{-1} \xi_{c}^{-2 n-1},
$$

where $\xi_{c}=c+\left(c^{2}-1\right)^{1 / 2}$ and $\left|\xi_{c}\right|>1$.

Example 4. Consider the Gaussian quadrature error

$$
E_{n}(f)=\int_{-1}^{1} w(x) \log (2-x) d x-\sum_{k=1}^{n} w_{k} \log \left(2-x_{k}\right)
$$

for $w(x)=(x+a)^{-1}, a=1.01$. Equations (9) and (14) give the estimate:

$$
\begin{aligned}
E_{n}(f) \approx & -4 \pi(3)^{1 / 2}\left[a+\left(a^{2}-1\right)^{1 / 2}\right]^{-1}\left[1+\left(a-\left(a^{2}-1\right)^{1 / 2}\right) / \xi_{c}\right]^{-2} \\
& \cdot(2 n+1)^{-1} \xi_{c}^{-2 n-1},
\end{aligned}
$$

where $\xi_{c}=2+3^{1 / 2}$. 
TABLE 4

Values for $\left|E_{n}(f)\right|$ in Example 4

\begin{tabular}{lll}
\hline$n$ & actual & estimated \\
\hline 3 & $.2069(-3)$ & $.1762(-3)$ \\
4 & $.1111(-4)$ & $.9839(-5)$ \\
5 & $.6373(-6)$ & $.5780(-6)$ \\
6 & $.3794(-7)$ & $.3511(-7)$ \\
\hline
\end{tabular}

Example 5. Choose $w(x)=\exp \left(-x^{2}\right)$ and $f(x)=\exp \left(x^{2}\right)$ for the following Gaussian quadrature:

$$
E_{n}(f)=\int_{-1}^{1} w(x) f(x) d x-\sum_{k=1}^{n} w_{k} f\left(x_{k}\right) .
$$

Again the abscissas $x_{k}$ and weights $w_{k}$ are available in [10]. In this case, $w^{\prime} / w=$ $-2 z$ is an entire function, therefore, Theorem 2 cannot be applied to estimate the function $D\left(\xi^{-1} ; w\right)$. Nevertheless, $D\left(\xi^{-1} ; w\right)$ can be evaluated exactly by $(5)$. We find that

$$
D\left(\xi^{-1} ; w\right)=\exp \left(-\frac{1}{2}-\frac{1}{2} \xi^{-2}\right)
$$

Applying the technique described in Case 1 for the entire function $f(x)=\exp \left(x^{2}\right)$, we obtain

$$
E_{n}(f) \approx(2 \pi)^{1 / 2}\left(h^{\prime \prime}\left(z_{0}\right)\right)^{-1 / 2} \xi_{0}^{-2 n-1} \exp \left(z_{0}^{2}-\frac{1}{2}-\frac{1}{2} \xi_{0}^{-2}\right)
$$

where

$$
\begin{gathered}
z_{0}=\left(2+2\left(4 n^{2}+4 n+2\right)^{1 / 2}\right)^{1 / 2} / 2 \\
h^{\prime \prime}\left(z_{0}\right)=2+8(2 n+1) z_{0}\left[-2+2\left(4 n^{2}+4 n+2\right)^{1 / 2}\right]^{3 / 2}
\end{gathered}
$$

and

$$
\xi_{0}=z_{0}+\left(z_{0}^{2}-1\right)^{1 / 2}
$$

In this example the other saddle point $-\left(2+2\left(4 n^{2}+4 n+2\right)^{1 / 2}\right)^{1 / 2} / 2$ also has a significant contribution to the contour integral and cannot be ignored. Therefore the estimated error consists of two terms:

$$
E_{n}(f) \approx \sum(2 \pi)^{1 / 2}\left(h^{\prime \prime}\left(z_{0}\right)\right)^{-1 / 2} \xi_{0}^{-2 n-1} \exp \left(z_{0}^{2}-\frac{1}{2}-\frac{1}{2} \xi_{0}^{-2}\right),
$$

where $z_{0}= \pm\left(2+2\left(4 n^{2}+4 n+2\right)^{1 / 2}\right)^{1 / 2} / 2$.

\section{TABLE 5}

Values for $\left|E_{n}(f)\right|$ in Example 5

\begin{tabular}{lll}
\hline$n$ & actual & estimated \\
\hline 3 & $.6839(-2)$ & $.6105(-2)$ \\
4 & $.4471(-3)$ & $.4734(-3)$ \\
5 & $.2295(-4)$ & $.2779(-4)$ \\
\hline
\end{tabular}


Acknowledgements. The author would like to thank Professor Frank G. Lether for his encouragement and direction during this work. Thanks are also due to the referee for pointing out the existence of [8] and [9].

Department of Mathematical Sciences

Alcorn State University

Lorman, Mississippi 39096

1. W. BARRETT, "On the convergence of sequences of rational approximations to analytic functions of a certain class," J. Inst. Math. Appl., v. 7, 1971, pp. 308-323.

2. M. M. Chawla \& M. K. JAIN, "Asymptotic error estimates for the Gauss quadrature formula," Math. Comp., v. 22, 1968, pp. 91-97.

3. P. DaVIS \& P. Rabinowitz, Methods of Numerical Integration, Academic Press, New York, 1975.

4. D. Elliotr, "The evaluation and estimation of the coefficients in the Chebyshev series expansion of a function," Math. Comp., v. 18, 1964, pp. 274-284.

5. G. FreUd, "Error estimates for Gauss-Jacobi quadrature formulae," Topics in Numerical Analysis (John J. H. Miller, Ed.), 1972, pp. 113-121.

6. I. S. Gradshteyn \& I. M. Ryzhix, Table of Integrals, Series and Products, Academic Press, New York, 1965.

7. V. I. KRYLOv, Approximate Calculation of Integrals, Macmillan, New York, 1962.

8. R. KUMAR, “A class of quadrature formulas," Math. Comp., v. 28, 1974, pp. 769-778.

9. R. Kumar, "Certain Gaussian quadratures," J. Inst. Math. Appl., v. 14, 1974, pp. 175-182.

10. R. Piessens \& M. Branders, Tables of Gaussian Quadrature Formulas, University of Leuven, 1975.

11. A. H. Stroud \& D. SeCrest, Gaussian Quadrature Formulas, Prentice-Hall, Englewood Cliffs, N. J., 1962.

12. G. Szegö, Orthogonal Polynomials, Amer. Math. Soc. Colloq. Publ., Vol. 23, Amer. Math. Soc., Providence, R. I., 1975.

13. B. VoN SYDow, "Error estimates for Gaussian quadrature formulas," Numer. Math., v. 29, 1977, pp. 59-64. 